\title{
A Comparison of Power-Efficient Broadcast Routing Algorithms
}

\author{
Intae Kang and Radha Poovendran \\ Department of Electrical Engineering, University of Washington, Seattle, WA 98195-2500 \\ email: \{kangit,radha\}@ee.washington.edu
}

\begin{abstract}
Following the seminal work of Wieselthier et al. on power-efficient broadcast routing, a novel technique called Embedded Wireless Multicast Advantage (EWMA) was proposed to further reduce the total transmit power of a broadcast routing tree. In our previous work, we showed that when the network lifetime is defined as the time for the first node failure due to battery depletion, the total transmit power is not the only measure of power-efficiency. We proved that either maximum transmit power or link longevity plays a crucial role in extending the network lifetime. In this paper, we compare the performance of four known power-efficient algorithms (and their variants) not only in terms of the total transmit power but also in terms of other performance measures such as static network lifetime, total receive and interference power, and maximum and average hop count which have direct impacts on physical, link, and MAC layers and on end-to-end network delay.
\end{abstract}

\section{INTRODUCTION}

Due to the broadcast nature of wireless medium for omnidirectional antenna, a unit of message sent to a receiver at the boundary of the transmission range reaches every node within the range for "free." Wieselthier et al. [1] coined a term "wireless broadcast (multicast) advantage" for this property. In [2], [11], it was shown that the construction of a broadcast routing tree with minimum total transmit power is NP-hard.

There are suboptimal greedy power-efficient tree constructing algorithms called Broadcast Incremental Power (BIP) [1] and Embedded Wireless Multicast Advantage (EWMA) [2]. In [1], [2], the performance of each algorithm was compared mainly in terms of the required total transmit power to construct a broadcast routing tree. Both BIP and EWMA cleverly make use of the wireless broadcast advantage in their tree construction algorithms. Other heuristics that further reduce the total transmit power after the construction of a routing tree were presented in [1]-[3], [9] as postsweep procedure in [1], [3] or perNodeMinimalize procedure in [9]. A common characteristic of the heuristics in [1] and [3] is the use of broadcast advantage to further reduce the total transmit power. We call this class of algorithms as inner postsweeping. This class of algorithms inspect and remove the redundant transmissions from a routing tree, thus reducing the total transmit power.

The EWMA algorithm in [2] is based on postsweeping as well. However, unlike the postsweep algorithm in [1], EWMA

This research was funded in part by NSF grant ANI-0093187 and ARO grant DAAD190210242. checks if an incremental increase in transmission power of a node can result in removal of other transmitting nodes leading to net reduction in total transmission power. We call this operation as outer postsweeping. Note that EWMA can be further refined as: $E W M A=(M S T)+($ inner postsweep $)+$ (outer postsweep), where MST stands for Minimum Weight Spanning Tree.

In our recent work [3], we showed that minimizing the total transmit power does not maximize the overall network lifetime, and presented an algorithm that maximizes the static network lifetime (MSNL). There are other important characteristics which affect the network lifetime. In this paper, we present performance comparison studies of these algorithms in terms of (i) the total transmit power, (ii) the static network lifetime (closely related to maximum transmit power), (iii) the total receive and interference power, (iv) hop count, and (v) the ratio of transmitting and receiving nodes.

An important idea behind power-aware routing [3], [5]-[9] is to incorporate the residual battery energy into routing decision process. Hence, to extend the network lifetime in broadcast routing, the link cost metrics should be designed to allow algorithms to adaptively assign proper transmit powers to nodes depending on the current battery energy and network topology.

The receive power is also an important performance measure because the receive power of the current generation of RF devices can take as much as a half of transmit power [14]. Therefore, the power consumption from the signal reception can significantly affect the network lifetime (e.g., especially in flooding). ${ }^{1}$ To analyze the effect of receive power, we introduce the terms physical and logical neighbor and show that the mismatch between them can be a good measure of wasted receive power. Since the receive and interference power at the receiver are determined by transmit power assignment, we can indeed analyze the impact of transmit power assignment of an algorithm on these quantities.

Interference by other transmitters decreases the signal-tonoise ratio (SNR) and therefore increases the bit-error-rate (BER) of the communications. An increased BER can significantly affect the overall energy-efficiency through channel contention in MAC layer and increased retransmission rate in the link layer. Hence, the estimation of the interference at a

\footnotetext{
${ }^{1}$ This indicates the importance of an intelligent dedicated radio hardware which can redirect the network traffic within the communication subsystem without having to go through CPU [14].
} 
receiver can be a good measure of the impact of an algorithm on BER performance.

The remainder of this paper is organized as follows. In the next section, we briefly discuss the network model. In Section III, we discuss additional performance measures. Section IV summarizes our simulation results and Section $\mathrm{V}$ concludes this paper.

\section{NETWORK MODEL}

We denote a network as a weighted directed graph $G=$ $(N, A)$ with a set $N$ of nodes and a set $A$ of directed edges (links), $A=\{(i, j)\}$. For a directed edge $(i, j) \in A$, let $\pi(j)$ denote the parent node of node $j$ (i.e., $\pi(j)=i$ ). Each node is labeled with a node ID $\in\{1,2, \ldots,|N|\}$.

The network connectivity in this paper is equivalent to the strong connectivity (or reachability) from the root (source) node. Furthermore, the link connectivity need not be bidirectional.

We assume that each node (host) is equipped with an omnidirectional antenna. The transmission power required to reach a node at a distance $d$ is proportional to $d^{\alpha}$ assuming that the proportionality constant is 1 for notational simplicity and $\alpha$ is the path loss (attenuation) factor that satisfies $2 \leq \alpha \leq 4$. To avoid the undue complication of notation, we also assume the receiver sensitivity threshold as $1(0 \mathrm{~dB})$.

Definition 1 (Static and Dynamic Network): (i) We define a static network as a wireless multihop adhoc network where underlying routing structure is not self-reconfigurable or does not change over time. (ii) When a wireless network is selfreconfigurable or changes over time, we will call it as dynamic network. We note that the term dynamic network does not imply network nodes are mobile. In this paper, the node locations are fixed or stationary.

The following RF and computational components [8] contribute to the battery energy drain:

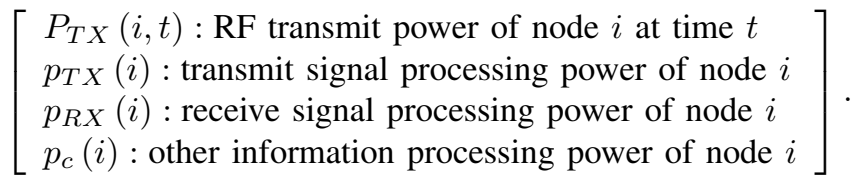

The RF component $P_{T X}(i, t)$ corresponds to the power consumption mainly due to power amplifier circuitry (PLL, $\mathrm{VCO}$, etc.) for transmission with an antenna. The other three components $p_{T X}(i), p_{R X}(i)$ and $p_{c}(i)$ are due to computational signal processing at node $i$. The transmit signal processing power $p_{T X}(i)$ is due to modulation, encoding and encryption, and the receive signal processing power, or simply receive power, $p_{R X}(i)$ is for corresponding inverse operations. $p_{c}(i)$ is related to all other signal processing power excluding communications at the node. We assume that computational components of every transceiver are same $\left(p_{T X}(i)=p_{T X}, p_{R X}(i)=p_{R X}\right.$ and $p_{c}(i)=p_{c}$ for all $i$ ).

\section{Performance Measures}

In this section, we present several important performance measures whose results will be compared by simulations in Section IV.

\section{A. Total Transmit Power}

Definition 2: Given a spanning tree $T$, the required pairwise transmit power $P_{i j}$ to maintain a link $(i, j) \in T$ from node $i$ to $j$ is $P_{i j}=d_{i j}^{\alpha}$ where $d_{i j}$ is the distance between the node $i$ and $j$. The actual (node) transmit power assigned to the node $i$ by the routing algorithm is

$$
P_{T X}(i)=\max _{j \in \Re_{i}}\left\{P_{i j}\right\}, \text { for } i \in N
$$

where $\Re_{i}$ is a set of adjacent (children) nodes of node $i$ in the tree.

Unlike conventional wired networks, there is no permanent connection between the nodes in wireless networks. The transmit power level $\left\{P_{T X}(i)\right\}$ assigned to each node $i$ (and node mobility, if it is a mobile adhoc network) determines the network topology.

Definition 3 (Physical and Logical Neighbor): If a node $i$ is transmitting with power $P_{T X}(i)$, then the physical neighbor $\aleph_{i}=\left\{k \mid 0<P_{i k} \leq P_{T X}(i)\right\}$ of node $i$ in a wireless network is the set of all the nodes within the communication boundary. The logical neighbor $\Re_{i}=\operatorname{adj}(i)=\{k \mid \pi(k)=i\}$ of node $i$ is the set of adjacent nodes in a routing tree.

In general, the physical neighbor determined by a network topology and (node) transmit power does not coincide with the logical neighbor determined by a routing algorithm. Note that $\aleph_{j}=\emptyset$ when $P_{T X}(j)=0$ and $\Re_{i} \subseteq \aleph_{i}$.

Assuming $d_{i k}>d_{i j}$, the incremental power $\Delta P_{j k}^{i}$ of node $i$ is defined as the additional power required to reach another node $k$ [1], i.e., $\Delta P_{j k}^{i}=P_{i k}-P_{i j}$. If we label every node in $\aleph_{i}$ as $i_{k}$ in an increasing order of distance from node $i$ (i.e., $i=i_{0}, i_{1}, \ldots, i_{\left|\aleph_{i}\right|}$ such that $P_{i i_{p}}<P_{i i_{q}}$ if $\left.p<q\right)$, then the transmit power $P_{T X}(i)$ of node $i$ can be represented as the sum of incremental power

$$
P_{T X}(i)=\sum_{k=0}^{\left|\aleph_{i}\right|-1} \Delta P_{i_{k} i_{k+1}}^{i} .
$$

Given a spanning tree $T$ with node $i$ transmitting with power $P_{T X}(i)$, the total transmit power of this tree is:

$$
\mathcal{P}_{T X}(T)=\sum_{i \in N} P_{T X}(i)=\sum_{i \in N} \sum_{k=0}^{\left|\aleph_{i}\right|-1} \Delta P_{i_{k} i_{k+1}}^{i} .
$$

We denote a tree with minimum total transmit power as

$$
\begin{aligned}
T^{\circ} & =\underset{T \subset G(N, A)}{\arg \min } \mathcal{P}_{T X}(T)=\underset{T \subset G(N, A)}{\arg \min } \sum_{i \in N} P_{T X}(i) \\
& =\underset{T \subset G(N, A)}{\arg \min } \sum_{i \in N} \sum_{k=0}^{\left|\aleph_{i}\right|-1} \Delta P_{i_{k} i_{k+1}}^{i} .
\end{aligned}
$$

Hence, the total transmit power is the same as the total incremental power. The BIP algorithm [1] effectively solves (5) 
to find a solution to (4). As noted earlier, finding an optimal solution to minimum total transmit power is NP-hard.

\section{B. Static Network Lifetime}

Definition 4 (Network Lifetime): Given a broadcast routing tree $T$, (i) the network lifetime $\mathcal{L}(T)$ is defined as the duration of the network operation until the first node failure due to battery depletion [7], assuming that broadcast from the source node starts with the network initialization. (ii) The static network lifetime refers to the lifetime when the routing tree $T$ does not change once the tree is setup at the initialization phase. (iii) The dynamic network lifetime refers to the case when the routing tree $T$ is updated based on an update policy (for example, either periodically or whenever there are changes in the network topology).

In this paper, we will concentrate on the static network case. Because the routing tree does not change over time, the transmit power $P_{T X}(i)$ is not a function of time. ${ }^{2}$ If the residual battery energy level of node $i$ at time $t$ is $E_{i}(t)$ and the node $i$ is using transmit power $P_{i j}$ to transmit to node $j$, this link can be maintained for the remaining $E_{i}(t) / P_{i j}$ units of time.

Definition 5 (Link and Node Longevity): We define the link longevity $l_{i j}$ of a link $(i, j) \in T$ as

$$
l_{i j}=\frac{E_{i}(0)}{P_{i j}} .
$$

The node longevity $\ell_{i}$ of a node $i$ is defined as follows:

$$
\ell_{i}=\min _{j \in \Re_{i}}\left\{l_{i j}\right\}=\frac{E_{i}(0)}{\max _{j \in \Re_{i}}\left\{P_{i j}\right\}}=\frac{E_{i}(0)}{P_{T X}(i)} .
$$

Both link and node longevity have time as their dimension. A node $i$ transmitting data with power $P_{T X}(i)$ can live for $\ell_{i}$ units of time. If a node $i$ is a leaf node in the spanning tree, then $P_{T X}(i)=0$ and thus $\ell_{i}=\infty$. Otherwise, the source and relay nodes have a finite node longevity.

Considering all the components introduced above, a realistic model of energy dissipation is

$$
\begin{aligned}
E_{i}(t) & =E_{i}(0)-\int_{0}^{t}\left[P_{T X}(i, \tau)+p_{T X}\right] I_{T}(i, \tau) d \tau \\
& -\int_{0}^{t} \sum_{j \in N} I_{R}(i, j, \tau) p_{R X} d \tau-\int_{0}^{t} p_{c} d \tau
\end{aligned}
$$

where $I_{T}(\cdot)$ and $I_{R}(\cdot)$ are indicator functions such that

$$
\begin{gathered}
I_{T}(i, t)= \begin{cases}1, & \text { if } i \text { is transmitting at time } t \\
0, & \text { otherwise }\end{cases} \\
I_{R}(i, j, t)= \begin{cases}1, & \text { if } i \in \aleph_{j} \text { at time } t \\
0, & \text { otherwise }\end{cases}
\end{gathered}
$$

We will call the sum of all node energies $\sum_{i \in N} E_{i}(t)$ at a given time $t$ as the energy pool of the network. As a special case, when the nodes of a network have identical initial energies (i.e.,

\footnotetext{
${ }^{2}$ Whenever the time-varing nature of transmit power needs to be emphasized, we will use the notation $P_{T X}(i, t)$.
}

$E_{i}(0)=\mathcal{E}$ for all $\left.i\right)$, we will denote the network as an equally distributed energy network (EDEN).

Note that in our battery model, we do not consider the nonlinear behavior of voltage as a function of remaining capacity [5] or the battery charge recovery effect due to diffusion process [12], [13], but use a simplified linear battery discharge model. These are left for future work.

Given an initial energy distribution $\left\{E_{i}(0)\right\}$ and the transmit power $\left\{P_{T X}(i)\right\}$, the static network lifetime of a tree $T$ is related to the link and node longevity as follows:

$$
\begin{aligned}
\mathcal{L}(T) & \equiv \min _{i \in N}\left\{\frac{E_{i}(0)}{P_{T X}(i)}\right\}=\min _{i \in N}\left\{\ell_{i}\right\} \\
& =\min _{i \in N}\left\{\min _{j \in \Re_{i}} l_{i j}\right\}=\min _{(i, j) \in A(T)}\left\{l_{i j}\right\},
\end{aligned}
$$

where $A(T)$ is the edge set induced by a tree $T$. Hence, the network lifetime of a tree $T$ is determined by a node with the minimum node longevity or a link with the minimum link longevity.

Definition 6: The (globally) optimal static network lifetime $\mathcal{L}^{*}$ is defined as

$$
\mathcal{L}^{*} \equiv \max _{T \subset G(N, A)}\{\mathcal{L}(T)\}=\max _{T \subset G(N, A)} \min _{(i, j) \in A(T)}\left\{l_{i j}\right\} .
$$

In [3], we showed that MSNL algorithm provides the optimal static network lifetime.

\section{Total Receive and Interference Power}

Given a routing tree structure, the source and relay nodes are transmitting nodes and the leaf nodes are receiving nodes.

Definition 7 (Total Receive Power): Let $p_{R X}(i)=p_{R X}$ for all $i$. The total receive power $\mathcal{P}_{R X}(T)$ of a network is

$$
\mathcal{P}_{R X}(T)=\sum_{j \in N} \sum_{i \in \aleph_{j}} p_{R X}(i)=p_{R X} \sum_{j \in N}\left|\aleph_{j}\right| .
$$

Because there are $(|N|-1)$ receivers in broadcasting, the only portion of receive power meaningfully processed by the receivers is $(|N|-1) p_{R X}$. Hence, the amount of wasted power due to unnecessarily processing the signal is

$$
\begin{aligned}
\mathcal{P}_{R X}^{\text {diff }}(T) & =\mathcal{P}_{R X}(T)-(|N|-1) p_{R X} \\
& =\sum_{j \in N}\left(\left|\aleph_{j}\right|-\left|\Re_{j}\right|\right) p_{R X}=\sum_{j \in N} \sum_{i \in \aleph_{j} \backslash \Re_{j}} p_{R X},
\end{aligned}
$$

where $\aleph_{j} \backslash \Re_{j}$ represents the set difference operation. When a node $i$ transmits with power $P_{T X}(i)$, the actual received power at node $j$ is $P_{T X}(i) / d_{i j}^{\alpha}$ due to channel attenuation.

Definition 8 (Total Interference Power): With a current transmit power assignment $\left\{P_{T X}(i)\right\}$, the total interference power at node $j$ is the sum of all received power:

$$
\mathcal{P}_{I}(T)=\sum_{i \in N \backslash\{j \cup \pi(j)\}} P_{T X}(i) / d_{i j}^{\alpha} .
$$

The received power whose signal strength is larger than the receiver threshold $(0 \mathrm{~dB}$ in this paper) will be detrimental 
against correct signal reception, since it is a data-like interference (or crosstalk). We will call this quantity, closely related to (11), as the total effective interference power

$$
\mathcal{P}_{I}^{e f f}(T)=\sum_{i \in N} \sum_{j \in \aleph_{i} \backslash \Re_{i}} \frac{P_{T X}(i)}{d_{i j}^{\alpha}} .
$$

The total effective interference power corresponds to cochannel interference and hence directly affects the BER performance in lower layers. Therefore, in designing a network routing algorithm, it is important to minimize the mismatch between physical and logical neighbors in order to reduce the total receive power as well as the total effective interference.

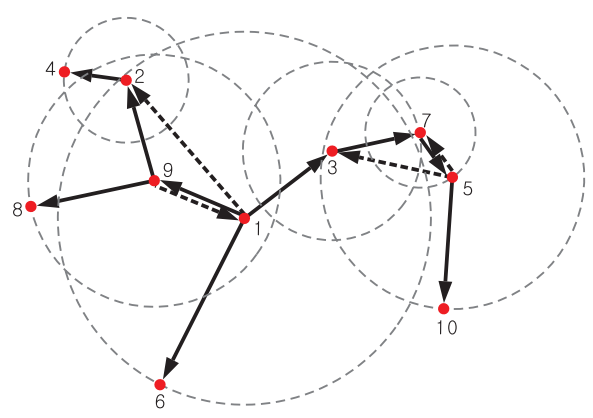

Fig. 1. Receive power and interference: dashed lines represent the mismatch between physical and logical neighbors.

Example 1: In Fig. 1, a sample broadcast routing tree for a network with 10 nodes is shown. The number of dashed lines (4, in this example) represents $\left(\left|\aleph_{j}\right|-\left|\Re_{j}\right|\right)$, and the amount of wasted receive power due to unnecessary signal processing (11) is $4 \cdot p_{R X}$ and total effective interference power (13) is $P_{T X}(1) / d_{12}^{\alpha}+P_{T X}(9) / d_{91}^{\alpha}+P_{T X}(5) / d_{53}^{\alpha}+P_{T X}(5) / d_{57}^{\alpha}$.

It should be noted that (12) and (13) is for the worst case scenario. For example, in Fig. 1, the transmission by each node is usually delayed by a small amount of propagation and processing delay. Hence, the total interference power is an approximation when there is a continuous broadcast traffic.

This clearly shows the impact of transmit power levels on the interference at the receiver node. The section IV presents the case study of this parameter for each of the algorithm. From these results, we can roughly estimate the BER performance of each routing algorithm.

\section{Hop Count-End-to-End Delay}

The number of hops is another important measure of performance, because the maximum hop count (network diameter) and average hop count are directly related to end-to-end delay. Moreover, if we assume equal probability of link failures, the smaller the number of hops, the more reliable the broadcast routing tree. Although we do not run packet level simulators such as ns- 2 or Glomosim, we can get an estimate of delay and reliability performance of the algorithms.

\section{Simulation Model and Results}

In this section, we perform simulations with the following model. Within a $1 \times 1 \mathrm{~km}^{2}$ square region, the network configurations (locations of nodes) are randomly generated according to uniform distribution. The same random seeds are used for valid comparison of each algorithm. $\alpha=2$ is used as a path loss factor. The initial energy $\left\{E_{i}(0)\right\}$ is distributed according to three uniform probability distributions: (i) unif $\left(10^{7}, 10^{7}\right)=$ constant, (ii) unif $\left(0.5 \times 10^{7}, 10^{7}\right)$, and (iii) unif $\left(0,10^{7}\right) .{ }^{3}$ Broadcast routing trees rooted at the source node (also located randomly in the grid) are constructed using various algorithms. We assume that a broadcast session initiates at time $t=0$ and carries a constant bit rate (CBR) traffic. The simulation results are for stationary (non-mobile) network topologies.

We append the suffix -SW for algorithms applied with inner postsweeping (e.g., MST vs. MSTSW). Each point in Fig. 2 represents an average of 100 different randomly generated network topologies. Note that in case of EDEN (undirected graph due to equal energy), MSNL exactly coincides with MST [3], and hence both curves perfectly overlap in Fig. 2. Also note that, except for the network lifetime, all other performance measures depend solely on network topology (not initial energy distribution) and hence only one curve for MST(-SW), BIP($\mathrm{SW})$ and EWMA is shown regardless of $\left\{E_{i}(0)\right\}$ in Fig. 2(b)(f).

In Fig. 2(a), the performance comparison in terms of total transmit power is shown. In general, the total transmit power of all trees decreases as network density increases. Hence, per node average transmit power will decrease even more rapidly. As presented in [2], EWMA (outer postsweeping), on average, performs best in terms of total transmit power. Because this measure is already well-studied in the previous literature [1], [2], we do not proceed into further details. However, what we need to observe is that both inner and outer postsweeping reduces the total transmit power and outer postsweeping provides a larger gain in lifetime.

Fig. 2(b) summarizes the lifetime performance of static trees for various distributions of the initial battery energy and the size of the networks $|N|$. Except for EWMA, the static network lifetime increases linearly as a function of the network size per $1 \times 1 \mathrm{~km}^{2}$ region, which is mainly due to increase in initial energy pool $\sum_{i \in N} E_{i}(0)$. On the other hand, in case of EWMA, there is almost no gain in lifetime regardless of initial energy distribution. As becomes clearer in Fig. 2(e) and 2(f), this is because EWMA relies on a smaller portion of nodes transmitting with a large transmit power. Note that EWMA starts from MST with inner postsweeping. Although transmissions from some nodes can be eliminated from MST by outer sweeping, this is achieved at the expense of increasing the transmit power of nodes. The maximum transmit power among the nodes, $\max _{i \in N}\left\{P_{T X}(i)\right\}$, becomes larger and hence this reduces the network lifetime. However, as shown in [3],

\footnotetext{
${ }^{3}$ unif $(\eta, \xi)$ denotes a uniform distribution ranging from the minimum value $\eta$ to the maximum value $\xi$, which represents the full battery capacity.
} 


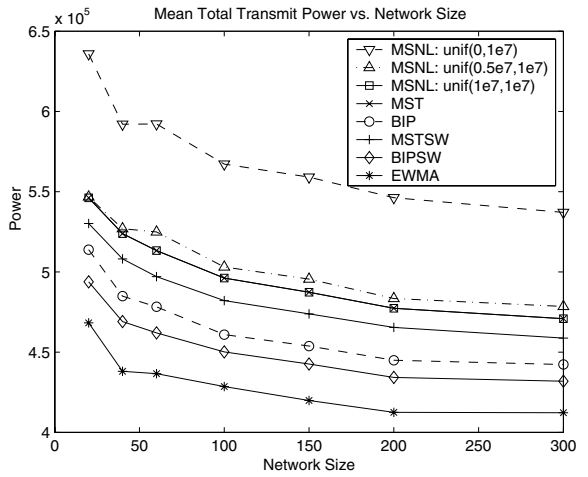

(a) Mean total transmit power

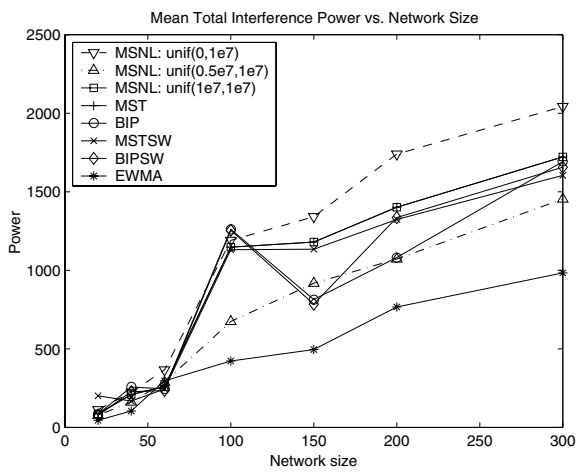

(d) Mean total effective interference power

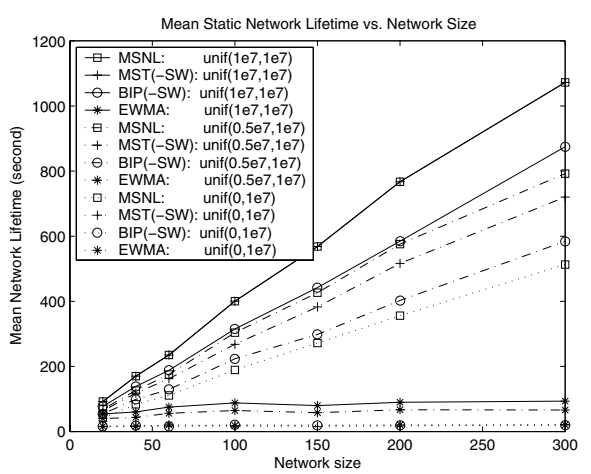

(b) Mean static network lifetime

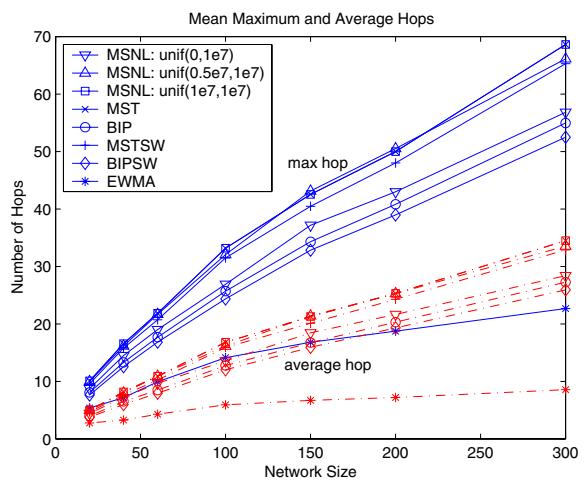

(e) Mean network diameter

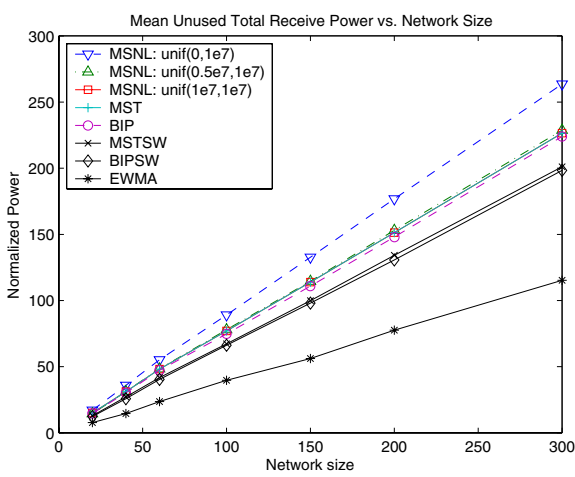

(c) Mean wasted total receive power

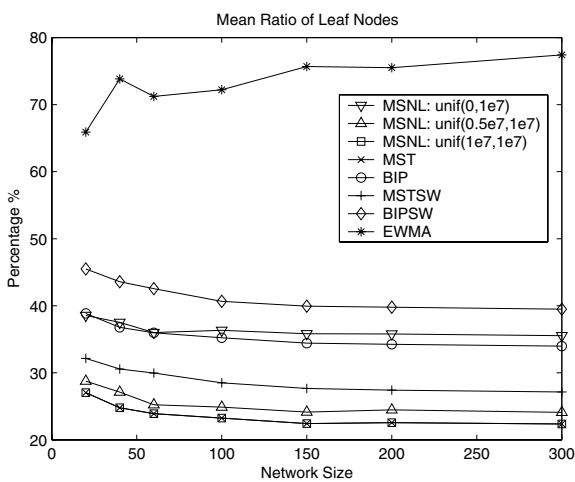

(f) Mean ratio of transmitting and receiving nodes

Fig. 2. Comparison of MSNL, MST, MSTSW, BIP, BIPSW, EWMA algorithms $(\alpha=2)$.

MSNL always produces longer lifetime. The separation of MSNL from other metrics becomes even more significant when $\left\{E_{i}(0)\right\}$ is unif $\left(0,10^{7}\right)$. This is because the max-min lifetime [7] is heavily dependent on the nodes with a scarce initial energy. MSNL adaptively trade-offs between network lifetime and total transmit power depending on the current residual battery energy.

Fig. 2(c) represents the number of mismatch between physical and logical neighbors $\mathcal{P}_{R X}^{\text {diff }}(T) / p_{R X}$ (the amount of normalized receive power wasted for unnecessarily processing the signal). The performances of MST and BIP and corresponding inner postswept version are almost identical, respectively. We suspect this is because the effect of broadcast advantage of BIP is comparable to the special property of MST, i.e., MST is a subset of relative neighborhood graphs (RNG) [15]. In RNG [15], it is guaranteed that no point lies within the lune region defined by two nodes incident on an edge in MST. Hence, this effectively reduces the unnecessary receive power. In MSNL with unif $\left(0,10^{7}\right)$ case, because the total transmit power is larger than the other cases and the link cost is non-Euclidean, it produces a larger mismatch between the logical and physical neighbors. We observe that inner and outer postsweeping also reduces the mismatch between physical and logical neighbors. EWMA performed best in this measure.

Fig. 2(d) shows the mean total effective interference power $\mathcal{P}_{I}^{e f f}(T)$ of each algorithm. Contrary to Fig. 2(c), the curves in this figure are quite irregular especially for BIP. In MST, more nodes are actively engaged in transmission, but with smaller transmission power. On the other hand, due to broadcast advantage, fewer nodes are actively transmitting in BIP trees but the transmission power of these nodes are larger. Therefore, it contains more number of leaf nodes than MST algorithm (hence, reducing the total transmit power). One observation is that minimal total transmit power does not necessarily imply the minimum transmit power at each node. We suspect this is one of the main causes of irregularity.

Fig. 2(e) and 2(f) compare the number of hops and ratio of leaf nodes, respectively. These two quantities are closely related to each other: if the ratio of leaf nodes is larger, smaller proportion of nodes are transmitting (with larger power) and hence the maximum and average hop counts become smaller. EWMA again produces best performance with smaller hop counts (end-to-end delay), since it tries to assign large transmit powers to nodes. Therefore, with respect to the parameters we have evaluated, EWMA seems to utilize the wireless broadcast advantage property most efficiently.

\section{CONCLUSiOnS}

In this paper, we presented an extensive comparative study of known power-efficient algorithms and heuristics. We compared not only the power-efficiency (total transmit power) but also other performance measures such as the static network lifetime, 
total receive and effective interference power, maximum and average hop counts and the ratio of transmitting (source and relay) and leaf nodes.

We observed that outer postsweep operation which is the core part of EWMA [2] has many favorable effects of reducing the total transmit, receive, and interference power and the number of hops, but at the cost of significantly reduced network lifetime. We believe that our simulation results provide insights that can help in developing improved heuristics trading-off different aspects of performance measures considered in this paper.

\section{REFERENCES}

[1] J.E. Wieselthier, G.D. Nguyen, and A. Ephremides. "On the Construction of Energy-Efficient Broadcast and Multicast Trees in Wireless Networks," Proc. IEEE INFOCOM 2000, pp. 586-594.

[2] M. Cagalj, Jean-Pierre Hubaux, C. Enz, "Minimum-energy broadcast in all-wireless networks: NP-completeness and distribution issues," MOBICOM'02, September, 2002, Atlanta, Georgia, USA.

[3] I. Kang and R. Poovendran, "Maximizing static network lifetime of wireless broadcast adhoc networks," IEEE ICC 2003, Alaska, USA

[4] A. Ephremides, "Energy concerns in wireless networks," IEEE Wireless Communications, vol. 9, issue: 4 , Aug 2002, pp. 48-59.

[5] S. Singh, M. Woo, and C.S. Raghavendra, "Power-aware routing in mobile ad hoc networks," Proc. ACM/IEEE MOBICOM, pp. 181-190, Dallas, TX, Oct. 1998.

[6] C.-K. Toh, "Maximum battery life routing to support ubiquitous mobile computing in wireless ad hoc networks," IEEE Communications Magazine, Jun. 2001, pp. 138- 147.

[7] J.H. Chang and L. Tassiulas, "Energy conserving routing in wireless adhoc networks," INFOCOM '2000, March 2000.

[8] V. Rodoplu and T. H. Meng, "Minimum energy mobile wireless networks," IEEE J. Selected Areas in Communications, vol. 17, Number 8, Aug. 1999.

[9] R. Ramanathan and R. Rosales-Hain, "Topology control of multihop wireless networks using transmit power adjustment," in Proc. IEEE INFOCOM 2000, March 2000.

[10] P.J. Wan, G. Calinescu, X. Y. Li, and O. Frieder, "Minimum-energy broadcast routing in static ad hoc wireless networks," Proc. IEEE INFOCOM 2001.

[11] A.E.F. Clementi, P. Crescenzi, P. Penna, G. Rossi, P. Vocca, "A Worstcase Analysis of an MST-based Heuristic to Construct Energy-Efficient Broadcast Trees in Wireless Networks," Technical Report 010 of the Univ. of Rome Vergata, 2001.

[12] M. Adamou, S. Sarkar, "A framework for optimal battery management for wireless nodes " Proceedings of INFOCOM 2002, pp. 1783-1792

[13] C.F. Chiasserini and R.R. Rao, "Energy efficient battery management," Proc. of Infocom 2000, Tel Aviv, Israel, March 2000.

[14] V. Raghunathan, C. Schurgers, Sung Park and M.B. Srivastava, "Energyaware wireless microsensor networks," IEEE Signal Processing Magazine, vol.19, issue: 2, March 2002, pp. 40 -50.

[15] G.T. Toussaint, "The relative neighborhood graph of a finite planar set," Pattern Recognition, vol.12, 1980, pp. 261-268. 